\title{
ОБЗОРЫ
}

\section{Влияние степени международной диверсификации бизнеса на его эффективность: существует ли оптимальный уровень международной диверсификации бизнеса?}

\author{
Щербаков Д.Ю. ${ }^{18}$
}

Данная статья содержит обзор исследований, посвященных анализу формы зависимости между уровнем международной диверсификаџии бизнеса и эффрективностью бизнеса. Показаны основные этапы эволючии идентифицируемых гипотез и используемых для этого количественных методов анализа. Приведены результаты ключевых исследований и дана их содержательная интерпретаџия: выделены этапы интернаџионализации бизнеса, каждый из которых характеризуется различным влиянием международной диверсификации на эфффективность бизнеса, сформулировань характеристики оптимального уровня интернационализации. Обобщение данных исследований позволяет сформулировать гипотезу о причинах существующих различий в эффрективности интернационализащии бизнеса на развитых и развивающихся рынках.

JEL: F23, F30, G30, G34

Ключевые слова: международная диверсификация бизнеса, эффективность бизнеса, интернационализаџия, транснащиональные корпораџии, продуктовая диверсификаџия

\section{Введение}

Последние два десятилетия характеризовались существенным темпом роста уровня интернационализации бизнеса - доли компаний, реализующих стратегии международной диверсификации. В частности, в 2000-х годах годовой темп роста количества международных сделок достигал $40 \%$ в отдельных географических общностях и индустриях ${ }^{19}$.

Тем не менее в большинстве исследований, посвященных вопросам эффективности международной диверсификации бизнеса, было показано, что международная диверсификация (далее - МД), как отдельный класс стратегий корпоративного развития, несет в себе существенный риск снижения эффективности бизнеса.

В данной работе представлен обзор исследований, посвященных анализу вопросов эффективности стратегий международной диверсификации бизнеса на основе бухгалтерских показателей эффективности (методология «accounting studies» ${ }^{20}$ ). Рассмотренные исследования позволяют ответить на вопрос о форме взаимосвязи между уровнем международной диверсификации бизнеса и его эффективностью. Следствием результатов данных исследований являются выводы относительно существования и характеристик оптимального уровня международной диверсификации бизнеса, отклонения от которого

\footnotetext{
${ }^{18}$ Магистр экономики Университета Сорбонна Париж-1 (2009), магистр экономики ГУ ВШЭ (2009), аспирант факультета экономики НИУ ВШЭ, младший менеджер отдела стратегии и операционной эффективности департамента бизнес-консультирования PricewaterhouseCoopers Russia B.V.

19 Данные приведены для промышленных отраслей Европейского союза. Источник: [Coeurdacier et al., 2009; Finkelstein, 1999].

20 Обзор не включает в себя исследования, проведенные по методологии «event-study» (анализ динамики акции в заданном временном интервале (окне) вокруг события, связанного с международной диверсификацией).
} 
могут снижать эффективность бизнеса.

Работа организована следующим образом: в первой части приводится краткая классификация основных мер степени международной диверсификации (интернационализации) бизнеса и критериев измерения эффективности интернационализации. Далее дается обзор результатов работ, посвященных исследованию формы взаимосвязи между степенью международной диверсификации бизнеса и его эффективностью, а также определяющих ее факторов. Приведены результаты исследований относительно характеристик оптимального уровня интернационализации бизнеса.

\section{Основные показатели уровня международной диверсификации бизнеса и критерии для измерения ее эффективности}

Повышение уровня международной диверсификации бизнеса возможно за счет как органического, так и неорганического роста на зарубежных рынках. В свою очередь неорганическое развитие классифицируется на международные сделки по слияниям и поглощениям, вступление в международные альянсы и организацию совместных транснациональных компаний. Большинство исследований, приводимых в данном обзоре, охватывают все перечисленные способы расширения на международных рынках, поскольку используют универсальные показатели, характеризующие степень международной диверсификации бизнеса.

\section{Показатели, измеряющие уровень международной диверсификации бизнеса}

В зависимости от исследовательских задач используются следующие показатели, характеризующие уровень интернационализации бизнеса:

- Масштаб диверсификации (scale) определяется показателями уровня вовлеченности деятельности компании в зарубежные операции (например, долей выручки зарубежных подразделений в общей выручке, а также аналогичными показателями долей операционной прибыли, штатной численности, стоимости зарубежных активов (см., например, работу Г. Киан и Д. Ли (Qian, Li, 2002)). В силу того что данные показатели не характеризуют географическую дифференциацию присутствия компании, использование данного класса показателей стало сочетаться с показателями охвата диверсификации.

- Охват диверсификации (scope) определяется как показатель географического разброса зарубежных операций компаний. Наиболее простой показатель данного класса количество стран, в которых компания имеет свои подразделения. Более информативными можно считать, например, показатель энтропии ${ }^{21}$, рассчитанный по количеству подразделений компании в каждой из стран, регионов. Еще более информативными являются показатели, учитывающие географическую отдаленность подразделений от страны дислокации головного подразделения.

Данные показатели масштаба и охвата в количественных моделях анализа могут включаться как в качестве двух отдельных переменных, так и с помощью интегральных индексов, в которые, наравне с перечисленными выше характеристиками, могут быть добавлены косвенные характеристики уровня диверсификации (например, отношение количества зарубежных собственников к общему количеству собственников; количество зарубежных фондовых площадок, на которых торгуются бумаги компании, пр. $)^{22}$.

\section{Меры эффективности международной диверсификации}

Результативность международной диверсификации в исследованиях, использующих

\footnotetext{
21 Данный показатель используется, например, в работе Г. Киан и Д. Ли.

22 Подробный обзор данных показателей можно найти, например, в работе М. Хитта (Hitt et al., 2006).
} 
бухгалтерские показатели эффективности бизнеса (accounting studies), обычно измеряется следующими показателями ${ }^{23}$ :

1. Показатели, полностью основанные на финансовой отчетности: выручка (Gugler et al., 2003), прибыль (Gugler et al., 2003), операционный денежный поток (Moeller and Schlingemann, 2004), показатели доходности ROS, ROE и ROA (Qian, Li, 2002), (Qian, 2002), EPS, показатели финансового рычага и ликвидности. Обычно данные исследования строятся на основе сравнения результатов компаний, изменивших уровень международной диверсификации, с результатами схожих компаний, не изменивших уровень международной диверсификации (см., например, исследование С. Моеллера и Ф. Шлингеманна). Перечисленные показатели позволяют проводить анализ результатов имплементации стратегий МД на длительных временных горизонтах (от года и более).

2. Комбинированные меры результативности, одновременно сочетающие в себе данные рыночной доходности акций и финансовых результатов компаний. Такими показателями являются, например, excess value ratio (EV), market-to-book ratio (MTB), price-to-earning-per-share (PE) (используются, например, в работе Г. Боднара (Bodnar et al., 2003)); Q Тобина (например, см. работу С. Чанга и С. Ванга (Chang, Wang, 2007)).

\section{Результаты исследований формы взаимосвязи между степенью международной диверсификации бизнеса и его эффективностью}

Начиная с 70-х годов прошлого века вопрос о функциональной форме зависимости эффективности бизнеса от уровня международной диверсификации является одним из наиболее обсуждаемых вопросов из области проблематики, связанной с международной диверсификацией бизнеса. На протяжении десятилетий вплоть до середины 1990-х годов основной тестируемой гипотезой был линейный вид данной зависимости. Однако в последние полтора десятилетия с ростом темпов интернационализации бизнеса и появлением больших массивов данных стало очевидно, что данная зависимость не является линейной, а форма зависимости может существенно различаться в зависимости от стран, отраслей, мотиваций компаний к интернационализации, а также используемых метрик для оценки эффективности бизнеса. Производным является вопрос о характеристиках оптимального уровня международной диверсификации.

На современном этапе исследований существующие результаты свидетельствуют о нелинейной форме зависимости, однако вид данной формы является предметом продолжающихся дискуссий: на различных выборках стран и при использовании различных метрик эффективности бизнеса получены как результаты, подтверждающие зависимости Uвида, так и обратную U-зависимость, а также зависимость S-вида.

\section{Нелинейная зависимость U-обратной формы}

Работа М. Хитта была одной из первых, в которых диагностирована нелинейная обратная U-зависимость между степенью интернационализации бизнеса (измеренная с использованием показателя энтропии, примененного к данным о выручке компаний) и его эффективностью (использовался показатель ROA). Данная зависимость была получена на основе оценки параметров нелинейного уравнения стандартными инструментами регрессионного анализа. Данное уравнение состоит из аддитивных компонент, одна из которых является квадратом основной объясняемой переменной, характеризующей степень международной диверсификации.

Обратная U-зависимость, однако, была диагностирована только для компаний с умеренным уровнем продуктовой диверсификации (см. рис. 1, пунктирная линия): с ростом

\footnotetext{
23 Обзор используемых методов измерения эффективности стратегий приведен также в главе 3 книги Р. Брюенера (Bruener, 2004).
} 
уровня международной диверсификации компании ее эффективность растет благодаря доступу к новым ресурсам и рынкам, отдачи от масштаба, однако после определенного предельного уровня начинает снижаться из-за возрастающих транзакционных издержек управления и координации. Однако для компаний с высокой продуктовой диверсификацией, a также монопродуктовых компаний выявленные зависимости имеют другие формы: положительную линейную и нелинейную U-форму соответственно. Автор объясняет это тем, что для получения положительного эффекта от международной диверсификации компания должна иметь опыт управления сложными многокомпонентными структурами: такой опыт, как правило, имеется у компаний с высоким уровнем продуктовой диверсификации, а при выходе на международные рынки данный опыт является востребованным и критичным для успеха международной диверсификации. Напротив, монопродуктовые компании не имеют такого опыта и при выходе на международные рынки должны инвестировать в построение соответствующих механизмов управления бизнесом; при появлении данного опыта, компания постепенно начинает достигать положительных результатов. Дополнительным фактором, влияющим на операционную эффективность бизнеса, является необходимость в дополнительном инвестировании в исследования, связанные с выходом на международные рынки - разработку продуктов и технологий.

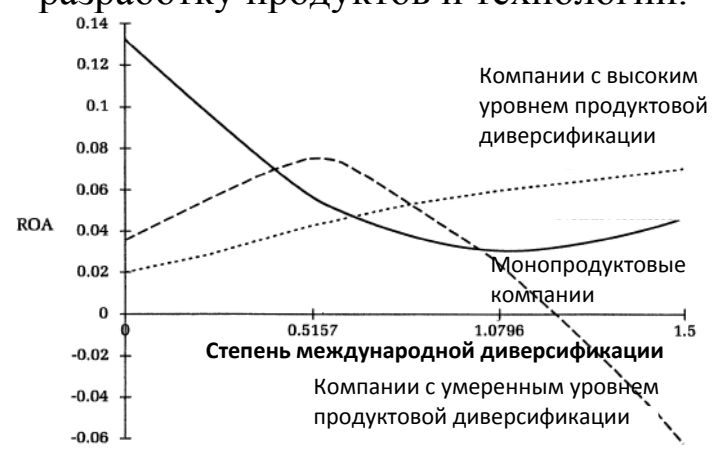

Рисунок 1. Результаты исследования М. Хитта: взаимосвязь МД и ROA

Анализ выявленной зависимости связи интернационализации и эффективности бизнеса от продуктовой диверсификации был продолжен, например, в работе С. Чанга и С. Ванга. Было показано, что форма обратной U-зависимости эндогенна относительно степени связанности продуктовой диверсификации компании: влияние интернационализации на эффективность компании тем выше, чем выше уровень связанной продуктовой диверсификации компании. Несвязанная диверсификация, наоборот, разрушает эффективность интернационализации. Автор объясняет данный эффект тем, что связанная продуктовая диверсификация по сравнению с несвязанной диверсификацией оказывает лучшее влияние на эффективность интернационализации по совокупности следующих пяти ключевых факторов: кросс-функциональное организационное обучение, синергии, механизмы внутренних контролей, способность адаптации внутренних установок к внешним условиям, издержки управления. 

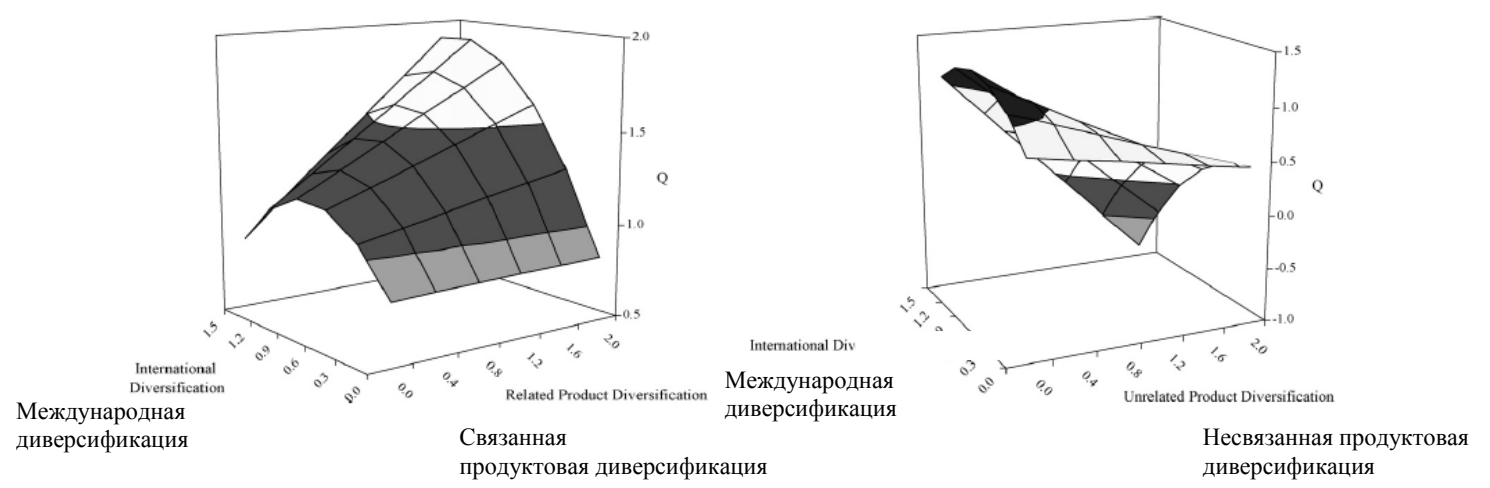

Рисунок 2. Результаты исследования С. Чанга и С. Ванга: функциональная аппроксимация зависимости Q Тобина от уровней международной и продуктовой диверсификаций (слева - для компаний сј связанной продуктовой диверсификацией, справа - для компаний с несвязанной диверсификацией)

\section{Нелинейная зависимость U-формы}

Наиболее частым результатом эмпирических исследований форм зависимости степени интернационализации и эффективности бизнеса является нелинейная U-форма. Данный результат получен, например, в работах Н. Капара и М. Котабе (Capar, Kotabe, 2003) для немецких компаний сферы услуг и В. Руигрока и X. Вагнера (Ruigrok, Wagner, 2003) для немецких промышленных компаний. Он объясняется тем, что с ростом международной диверсификации компании изначально высокие предельные издержки управления начинают компенсироваться выигрышами от обучения, и, начиная с определенного момента («дна»), создавать положительные эффекты от МД. В качестве меры эффективности в работе В. Руигрока и X. Вагнера рассматривает ROA и отношение операционных затрат к выручке (см. рис 3). Для обоих показателей демонстрируются одинаковые тенденции к повышению эффективности бизнеса с ростом опыта международной диверсификации. Тем не менее отмечается, что данный результат является характерным для развитого рынка Германии и может отличаться для других стран и рынков.
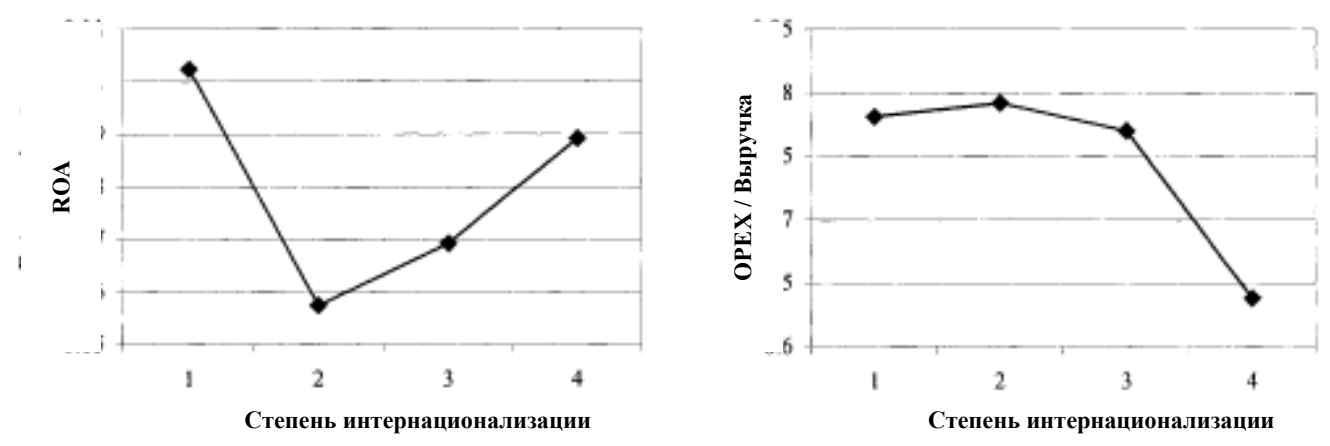

Рисунок 3. Результаты исследования В. Руигрока и Х. Вагнера: зависимость между эффективностью и уровнем интернационализации имеет U-форму

В работе Ф. Контрактора (Contractor et al., 2007) проведено исследование компаний одного из развивающихся рынков (Индия). В данном исследовании также диагностирована $\mathrm{U}$-зависимость, дифференцирующаяся по секторам экономики: компании сектора услуг проходят «дно» быстрее, чем промышленные предприятия. U-форма взаимосвязи интерпретируется как последовательное двухэтапное изменение соотношений выгод и издержек от интернационализации:

- на начальном этапе выхода на международные рынки издержки, связанные с 
интернационализацией (издержки получения знаний, координации и контроля удаленных подразделений, прочие транзакционные издержки) высоки по сравнению с малой базой выпуска, на которую они распределяются,

- на этапе зрелости международной диверсификации компании выгоды от интернационализации бизнеса (экономия от масштаба и охвата, диверсификация национальных рисков, доступ к экономичным ресурсам, доступ к знаниям, рост рыночной власти, пр.) превышают транзакционные издержки.

Ф. Контрактор показывает, что для компаний с развивающихся рынков третий этап, дополняющий U-форму до S-формы и характеризующийся избыточным уровнем МД (при котором географически диверсифицированные холдинги становятся слабоуправляемыми), не являются характерными в силу, как правило, недостаточно большого размера корпораций.

\section{Нелинейная зависимость S-формы}

Последней волной исследований в области связи эффективность-интернационализация стала волна исследований, идентифицирующих горизонтальную S-форму связи. Ее отличием от U-формы является наличие третьего этапа снижающейся эффективности от избыточной МД.

К работам последней волны исследования S-формы взаимосвязи относятся исследования Д. Лу и П. Беамиш (Lu, Beamish, 2004), А. Бобилло (Bobillo et al., 2010), A. Ругмана и X. Чанга (Rugman, Chang, 2010). В работах проводится параметрическая оценка полинома третьей степени и диагностируется взаимосвязь $\mathrm{S}$-вида по данным о транснациональных компаниях с развитых рынков.

В дополнение к детерминантам эффективности МД, идентифицированных в предыдущих исследованиях, в данных работах выделен показатель объема инвестиций в нематериальные специфичные активы интернационализирующихся компаний. По мнению авторов, данные активы могут приносить повышенную доходность с ростом международной диверсификации компании.
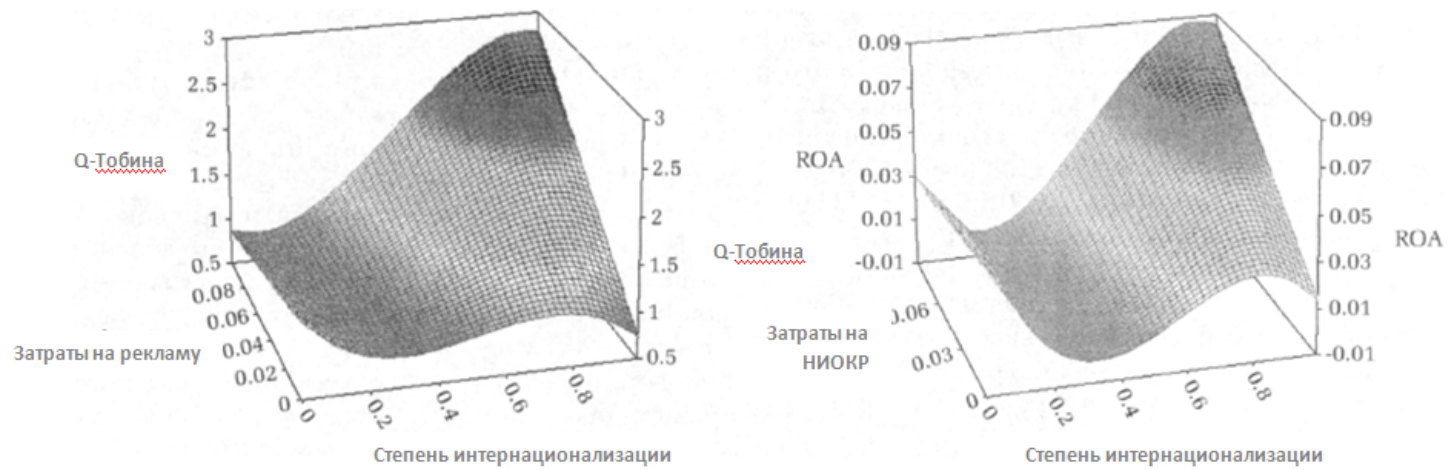

Рисунок 4. Результаты исследования Д. Лу и Р. Беамиш: диагностирована S-форма зависимости эффективность-интернационализация

Также необходимо выделить новую волну работ, использующих альтернативный инструментарий оценки зависимости интернационализация-эффективность, например метод нейронных сетей, использованный Д. Гарбе и Н. Рихтер (Garbe, Richter, 2009). Также перспективным направлением исследований является использование инструментов непараметрической эконометрики, не накладывающих изначальных функциональных ограничений на форму взаимосвязи.

\section{Характеристики оптимального уровня интернационализации бизнеса}

Вопрос о существовании и характеристиках оптимального уровня международной 
диверсификации бизнеса является производным от формы взаимосвязи между уровнем МД и эффективностью бизнеса. Наиболее общей формой взаимосвязи является S-форма. Она предполагает наличие одного максимума зависимости эффективность-интернационализация (переход от этапа зрелости МД к этапу избыточной МД), отклонение от которого по показателю уровня МД приводит к снижению эффективности бизнеса. Показано, что интернационализирующиеся компании с развивающихся рынков могут не достигать данного уровня МД, после которого дальнейшая интернационализация является убыточной (не достигается критический размер зарубежного бизнеса корпорации, при котором транзакционные издержки управления и контроля превышают выгоды от МД). Данная предпосылка может являться одним из объяснений факта большей эффективности сделок по международным слияниям и поглощениям компаний с развивающихся рынков, нежели компаний с развитых рынков. На оптимальный уровень МД также влияют такие факторы, как степень и тип продуктовой диверсификации, инвестиции в нематериальные активы и другие характеристики компании (такие как характеристики структуры собственников, совета директоров и правления, организационной структуры и пр.), а также ее окружающей среды (например, характеристики институциональной среды, конкурентной среды в отрасли, уровень государственного регулирования, доступные ресурсы в странах присутствия и пр.) $)^{24}$, определяющие соотношение выгод и издержек от международной диверсификации.

\section{Заключение}

В данной работе дан краткий обзор части исследований, использующих бухгалтерские показатели эффективности бизнеса (accounting studies), посвященных вопросам эффективности международной диверсификации бизнеса. Даны характеристики основных мер степени интернационализации бизнеса, а также мер эффективности бизнеса, используемых в исследованиях данного класса. Приведены результаты выборочных исследований относительно формы взаимосвязи интернационализация-эффективность. Показано, что идентифицируемые гипотезы последовательно эволюционировали от линейной формы зависимости эффективность-интернационализация к нелинейной обратной U-зависимости, зависимости U-формы, и далее к зависимости горизонтальной S-формы. B последнее время большую актуальность приобретают исследования, использующие более продвинутый инструментарий анализа, изначально не накладывающий функциональных ограничений на вид зависимости. Большинство исследований диагностируют существование оптимального уровня МД для компаний с развитых рынков капитала, в то время как основная масса компаний с развивающихся рынков еще не достигла того уровня избыточной международной диверсификации, после которого транзакционные издержки начинают превышать потенциальные выгоды от интернационализации.

\section{Список литературы}

1. Ивашковская И.В., Шамраева С.А. Эффективность международных сделок слияний и поглощений компаний с развивающихся рынков капитала: эмпирический анализ, // Материалы докладов Научной конференции ГУ-ВШЭ (апрель 2010 г.).

2. Aggarwal, R., and Aung, K.N. (2010), Capital structure, dividend policy, and multinationality: Theory versus empirical evidence, International Review of Financial Analysis, 19 (2010) 140-150.

3. Bertoni, F., Elia, S., and Rabbiosi, L. (2009), Drivers of acquisitions from BRICs to advanced countries: firm-level evidence, Copenhagen Conference on: Emerging Multinationals (2009).

4. Bobillo, A.M., Lopez-Iturriaga, F., and Tejerina-Gaite, F. (2010), Firm performance and international diversification: The internal and external competitive advantages, International

\footnotetext{
${ }^{24}$ Перечень данных факторов приведен, например, в работе М. Хитта.
} 
Business Review, 19 (2010) 607-618.

5. Bodnar, G., Tang, C., and Weintrop, J. (2003), The Value of Corporate International Diversification // NBER Working Paper, 2003.

6. Borde, S.F., Whyte, A.M., Wiant, K.J., and Hoffman, L.L. (1998), New evidence on factors that influence the wealth effects of international joint ventures, Journal of Multinational Financial Management, 8 (1998) 63-77.

7. Bruener, R.F. (2004), Applied Mergers and Acquisitions - John Wiley \& Sons, New Jersey.

8. Capar, N., and Kotabe. M. (2003), The relationship between international diversification and performance in service firms, Journal of International Business Studies, 34 (2003), 345355.

9. Carpenter, M.A., Pollock, T.G. and Leary, M.M. (2003), Testing a Model of Reasoned RiskTaking: Governance, the Experience of Principals and Agents, and Global Strategy in HighTechnology IPO Firms, Strategic Management Journal, 24 (2003) 803-820.

10. Chang, S., and Wang, C. (2007), The effect of product diversification strategies on the relationship between international diversification and firm performance, Journal of World Business, 42 (2007) 61-79.

11. Chittoor, R., and Ray, S. (2007), Internationalization paths of Indian pharmaceutical firms - A strategic group analysis, Journal of International Management, 13 (2007) 338-355.

12. Chkir I.E., and Cosset J.-C. (2001), Diversification strategy and capital structure of multinational corporations, Journal of Multinational Financial Management, 11 (2001) 1737.

13. Coeurdacier N., De Santis R.A. and Aviat A. (2009), Cross-Border Mergers and Acquisitions: Financial and Institutional Forces, Working Paper Series, no 1018 (March 2009) European Central Bank

14. Collins, J., Holcomb, T., Certo, S., Hitt, M., and Lester, R. (2009), Learning by doing: Cross-border mergers and acquisitions, Journal of Business Research, 62 (2009) 1329-1334.

15. Contractor, F.J., Kumar, V., and Kundu, S.K. (2007), Nature of the relationship between international expansion and performance: The case of emerging market firms, Journal of World Business, 42 (2007) 401-417.

16. Cuervo-Cazurra, A. (2007), Sequence of value-added activities in the multinationalization of developing country firms, Journal of International Management, 13 (2007) 258-277.

17.Denis, D.J., Denis D.K., and Yost K. (2002), Global Diversification, Industrial Diversification and Firm Value, The Journal of Finance, LVII (5) ( 2002) .

18. Dos, S.M.B., Errunzab, V.R., and Millerc, D.P. (2008), Does corporate international diversification destroy value? Evidence from cross-border mergers and acquisitions, Journal of Banking \& Finance, 32 (12) (2008) 2716-2724.

19. Finkelstein, S. (1999), Cross-Border Mergers and Acquisitions, Financial Times Mastering Global Business: The Complete MBA Companion in Global Business, (1999) 119-123.

20. Fridolfsson, S.-O. and Stennek, J. (2003), Why Mergers Reduce Profits and Raise Share Prices-A Theory of Preemptive Mergers, Journal of the European Economic Association, 3(5) (2005)1083-1104.

21. Garbe, J.-N., and Richter, N.F. (2009), Causal analysis of the internationalization and performance relationship based on neural networks - advocating the transnational structure, Journal of International Management, 15 (2009) 413-431.

22. Garcia-Canal, E., and Sanchez-Lorda, P. (2007), One more only if it is one of us. The number of partners and the stock market reaction to domestic and international alliance formation in EU telecom firms, International Business Review, 16 (2007) 83-108.

23. Gerpott, T.J., and Jakopin, N.M. (2007), Firm and target country characteristics as factors explaining wealth creation from international expansion moves of mobile network operators, Telecommunications Policy, 31 (2007) 72-92.

24. Gugler, K., Mueller. D.C., Yurtoglu. B. B., and Zulehner, C. (2003), The effects of mergers: an international comparison, International Journal of Industrial Organization, 21 (2003) 
625-653.

25.Herrmann, D.R., Hope, O.-K., and Wayne, B.T. (2008), International Diversification and Forecast Optimism: The Effects of Reg FD, Accounting Horizons Vol. 22, No. 2 American Accounting Association, (2008) 179-197.

26. Hitt, M., Hoskisson, R., and KimInternational, H. (1997), International Diversification: Effects on Innovation and Firm Performance in Product-Diversified Firms, The Academy of Management Journal, 40(4) (1997) 767-798.

27. Hitt, M.A., Tihanyi, L., Miller, T., and Connelly, B. (2006), International Diversification: Antecedents, Outcomes, and Moderators, Journal of Management, 32 (6) (2006) 831-867.

28. Joliet, R., and Hubner, G. (2006), Corporate International Diversification and the Cost of Equity: European Evidence, Working paper of Ecole de Gestion de l'Universite de Liege \#200605/01.

29. Kiymaza, H., and Mukherjeeb, T.K. (2001), Parameter shifts when measuring wealth effects in cross-border mergers, Global Finance Journal, 12 (2001) 249-266.

30. Lee, J.Y., Tang, C. and Tikoo, S. (2006), The Degree of Internationalization and the Stock Market Valuation of Earnings, Advances in International Accounting, 19 (2004) 201-219.

31. Lu, J.W., and Beamish, P.W. (2004), International Diversification and Firm Performance: The S-Curve Hypothesis, The Academy of Management Journal, 47(4) (2004) 598-609.

32. Moeller, S., and Schlingemann, F. (2004), Are cross-border acquisitions different from domestic acquisitions? Evidence on stock and operating performance for U.S. acquirers, Journal of Banking and Finance, (2004).

33. Olibe, K.O., Michello, F.A., and Thorne, J. (2008), Systematic risk and international diversification: An empirical perspective, International Review of Financial Analysis, 17 (2008) 681-698.

34. Park, M.-C., Yang, D.-H., Nam, C., and Ha, Y.-W. (2002), Mergers and Acquisitions in the Telecommunications Industry: Myths and Reality, ETRI Journal, 24(1) (2002) 56-68.

35. Qian, G. (2002), Multinationality, product diversification, and profitability of emerging US small- and medium-sized enterprises, Journal of Business Venturing, 17 (2002) 611-633.

36. Qian, G., and Li, J. (2002), Multinationality, global market diversification and profitability among the largest US firms, Journal of Business Research, 55 (2002) 325-335.

37. Rugman, A. M., and Oh, C. H. (2010), Does the regional nature of multinationals affect the multinationality and performance relationship? International Business Review, 19 (2010) 479-488.

38. Ruigrok, W., and Wagner, H. (2003), Internationalization and Performance: An Organizational Learning Perspective Management International Review, 43 (2003) 63-83.

39. Sánchez-Lorda, P. (2006), Stock Market Response to Acquisitions and Alliances in the European Telecom Industry: An Information Asymmetry Perspective, ETRI Journal, 28( 5) (2006).

40. Siah, H.A. (2007), International diversification, Business Review (University of Auckland),.9(1) (2007).

41. Staikouras, S.K. (2009), An event study analysis of international ventures between banks and insurance firms, International Financial Markets, Institutions and Money, 19 (2009) 675-691.

42. Verbeke, A., and Brugman, P. (2009), Triple-testing the quality of multinationalityperformance research: An internalization theory perspective, International Business Review, 18 (2009) 265-275.

43. Wang, S.-F., and Liao, C.-C. (2009), Cross-border Takeovers: Western Europe Evidence // 17th Conference on the Theories and Practices of Securities and Financial Markets, (2009).

44. Wiersema, M.F., and Bowen, H.P. (2008), Corporate diversification: the impact of foreign competition, industry globalization, and product diversification, Strategic Management Journal, 29(2) (2008) 115-227. 